\title{
Novel biosensor for detecting Hemoglobin and its oxidation state based on nonreciprocity in a coupled waveguide system
}

\author{
Shourya Dutta-Gupta*a ${ }^{*}$ Olivier J. F. Martin ${ }^{\mathrm{a}}$, and S. Dutta Gupta ${ }^{\mathrm{b}}$ \\ ${ }^{a}$ Swiss Federal Institute of Technology Lausanne, Lausanne CH-1015, Switzerland; \\ ${ }^{\mathrm{b}}$ School of Physics, University of Hyderabad, Hyderabad 500046, India; \\ * shourya.duttagupta@epfl.ch; phone +41216937864; fax +41216932614; www.nanophotonics.ch
}

\begin{abstract}
We study the reflection of a tightly focused Gaussian beam off a near symmetric resonant tunneling structure comprising two identical coupled waveguides. The coupled waveguides are loaded on each side by a spacer layer and a high index prism. Reflection of a Gaussian beam from such a resonant structure is associated with beam distortion and even beam splitting. We start with the distortion of the beam profile for a symmetric structure as a function of various parameters of the system. The broken spatial symmetry is introduced through the reference channel on one side and the sample channel on the other side as spacer layers. We monitor the dip in the beam profile when the two channels are filled with the sample and the reference liquid. We show that presence and absence of hemoglobin and its oxygenation states can be quantified by looking at the beam profile dip. Our results may find applications in high resolution sensing.
\end{abstract}

Keywords: resonant tunneling, coupled waveguides, Hemoglobin, Goos-Hänchen shift.

\section{INTRODUCTION}

There has been a great deal of interest in sensing using the surface and guided modes of a stratified medium. Usually such sensors make use of the attenuated total reflection dip, which heralds the excitation of the surface/guided mode [13]. The angular or the spectral location of the dip is extremely sensitive to any perturbation layer (its refractive index, width, etc...). Thus the shift of the dip gives a quantitative measure of the properties of the perturbing layer. Recently it was shown by a Stanford group that phase sensitive measurement can prove to be more accurate [4]. Goos-Hänchen (GH) shift of a Gaussian beam associated with reflection at an interface carries the required phase information [5- 6]. It was shown that the GH shift in a geometry supporting a surface plasmon can be used to enhance the accuracy by one or two orders of magnitude [4]. The phase singularity at the surface plasmon resonance is responsible for the enhanced sensitivity. A simple-minded stationary phase approximation [6] was used to predict the GH shift, though there have been reports on how the stationary phase approximation breaks down as a consequence of the sharp change in phase near a resonance. Thus a more rigorous method based on angular spectrum decomposition is needed to analyze such structures. There have also been reports on temperature sensing and other sensing devices making use of GH shift [7- 8]. Very recently a coupled waveguide structure with slight asymmetry was investigated to show GH shift with opposite signs for illumination from opposite ends [9]. A resonant tunneling geometry (see Fig. 1) was chosen, which allowed transmission only when the coupled modes were excited. Resonant tunneling geometry have found applications in many other contexts involving slow and fast light, cavity QED and even metamaterials [10- 12]. The asymmetry reported in Ref. [9] on sign reversal of the GH shift was a consequence of the non-reciprocity relations derived earlier [13-14]. The non-reciprocity in a lossless asymmetric structure is expressed through the non-reciprocal phases and hence nonreciprocal GH shift, while the intensity reflection is identical from both ends. Addition of absorption makes the reflection coefficient non-reciprocal as well. In this paper we introduce the spatial asymmetry through non identical spacer layers, say layer 1 is used as a sample channel while layer 5 is used as the reference channel. Moreover, we do not use the usual GH shift magnitude as in Ref. [4], rather, we use the minimum of the beam profile which results when a tightly focused beam shifts in reflection from a resonant structure. Let us stress that the distorted beam contains information about the

Plasmonics in Biology and Medicine IX, edited by Tuan Vo-Dinh, Joseph R. Lakowicz, Proc. of SPIE Vol. 8234, 82341C · @ 2012 SPIE · CCC code: 1605-7422/12/\$18 · doi: 10.1117/12.907859 
amplitude and phase of the complex reflection coefficient. Such Gaussian beam splittings have been reported in literature $[9,15]$ and observed in experiment [16].

The structure of the paper is as follows. In section 2, we define our system and geometry of excitation for both forward and backward illuminations. We also present a brief discussion on the dielectric response of Hemoglobin in both oxygenated and deoxygenated states. The next section contains the results of our numerical study for the near symmetric structure. For comparison we present the results for the symmetric structure also. Finally, in the last section we summarize our main results.

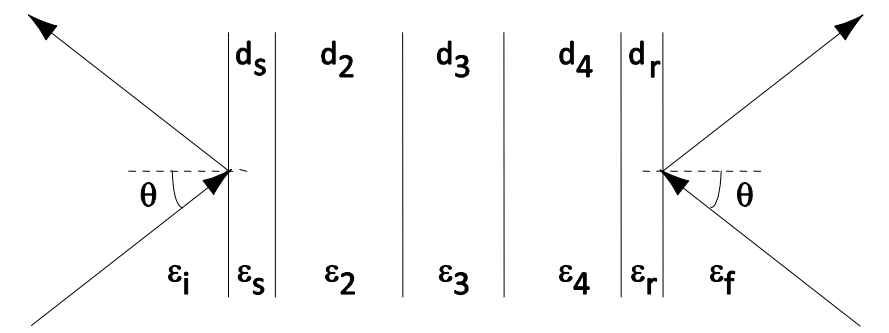

Figure 1. Schematics of the resonant tunneling geometry.

\section{FORMULATION OF THE PROBLEM}

Consider a resonant tunneling structure as shown in Fig.1. Two waveguides with widths $d_{2}$ and $d_{4}$ and dielectric functions $\varepsilon_{2}$ and $\varepsilon_{4}$, respectively, are separated by a spacer layer of thickness $\mathrm{d}_{3}$ and dielectric function $\varepsilon_{3}$. On either side of the waveguides two nano-channels are placed, one acting as the sample (with width $\mathrm{d}_{\mathrm{s}}$ and dielectric function $\varepsilon_{\mathrm{s}}$ ) and the other as the reference (with width and dielectric function given by $\mathrm{d}_{\mathrm{r}}$ and $\varepsilon_{\mathrm{r}}$ ) respectively. The layered medium is placed in between two high index prisms. Except for the first and the fifth layer the structure is assumed to be symmetric. The system can be illuminated by a plane, say, a TE-polarized wave at an angle $\theta$ from left (right), henceforth referred to as forward (backward) illumination and labeled by subscript $f(b)$.

It is well understood that there is a one to one correspondence between the Helmholtz equation in optics and the one dimensional Schrödinger equation. Thus the resonant tunneling structure is analogous to two coupled wells separated by a barrier. Transmission through such a system can be realized only via the excitation of the discrete modes of the guides. As mentioned earlier such structures have been studied in detail in other contexts [10-12]. Our interest in this structure is motivated by a somewhat different goal. Coupled waveguide structure for adequate coupling strength is bound to support coupled modes. The separation between the resonance dips of the coupled modes can be easily controlled via the spacer layer thickness between the two guides. Thus such a structure offers the flexibility where the resonances and their widths can easily be controlled. Furthermore, the widths of the reference and the sample channels and the refractive indices of the channel liquids control the excitation efficiency of the modes.

The dielectric function of Hemoglobin was modeled using the following equation,

$$
\begin{aligned}
& \varepsilon_{\text {oxy }, \text { deoxy }}=\varepsilon_{w}+\sum_{i=1}^{3} \frac{f_{p i}^{2}}{f_{0 i}^{2}-f^{2}-i \gamma_{i} f}, \\
& \varepsilon_{\text {eff }}=f_{r} \varepsilon_{o x y}+\left(1-f_{r}\right) \varepsilon_{\text {deoxy }}
\end{aligned}
$$

where the parameters $f_{p i}, f_{o i}$ and $\gamma_{i}$ represent the resonance strength, frequency and damping respectively of the different resonances. $f$ is the frequency and $\varepsilon_{\mathrm{w}}$ is the dielectric constant of water $\left(\varepsilon_{\mathrm{w}}=1.7689\right)$. For oxygenated (deoxygenated) Hemoglobin the Q-band(s) are at $541 \mathrm{~nm}$ and $570 \mathrm{~nm}(556 \mathrm{~nm})$. For a partly oxidized solution of Hemoglobin we use a linear combination of the dielectric functions of oxygenated and deoxygenated Hemoglobin as given in Eq. 1, where $f_{r}$ is the fraction of oxygenated Hemoglobin. If $f_{r}$ is equal to 1 then the solution is completely oxygenated and when $f_{r}$ is equal to 0 then it is completely deoxygenated. It should be noted that the parameter $f_{p i}$ is dependent on the concentration of Hemoglobin present.

In the next section, we first show the coupled mode resonances and their dependence on the system parameters. And finally we present out results as regards the sensing ability of a near symmetric structure. 


\section{RESULTS AND DISCUSSIONS}

We first look at a symmetric structure where we have used the following system parameters: both the sample and the reference layer were taken to be water with $\varepsilon_{\mathrm{s}}=\varepsilon_{\mathrm{r}}=1.7689$ and $\mathrm{d}_{\mathrm{s}}=\mathrm{d}_{\mathrm{r}}=0.1 \mu \mathrm{m}$. Other parameters were chosen as: $\lambda_{0}=0.5770 \mu \mathrm{m}, \varepsilon_{2}=\varepsilon_{4}=3.9085, \mathrm{~d}_{2}=\mathrm{d}_{4}=0.5 * \lambda_{0} /\left(\varepsilon_{2}\right)^{0.5}=0.1459 \mu \mathrm{m}, \varepsilon_{3}=2.2883$ and $\varepsilon_{\mathrm{i}}=\varepsilon_{\mathrm{f}}=6.145$. The incident wavelength $\lambda_{0}$ is chosen such that it coincides with one of the Q-bands of oxygenated Hemoglobin (at $0.5770 \mu \mathrm{m}$ ) [17]. We first study the dispersion characteristics assuming a plane wave illumination with a goal to delineate the under coupled, critically coupled (with a flat dip in the reflectivity profile) and over coupled (with distinct split resonances) regimes. The results are shown in Fig. 2., where we have plotted the intensity reflection coefficient as a function of angle of incidence and the spacer layer thickness. As can be clearly seen from Fig. 2 the aforesaid regimes are characterized by $d_{3}>0.2795 \mu \mathrm{m}, \mathrm{d}_{3}$ $\sim 0.2795 \mu \mathrm{m}$ and $\mathrm{d}_{3}<0.2795 \mu \mathrm{m}$, respectively.

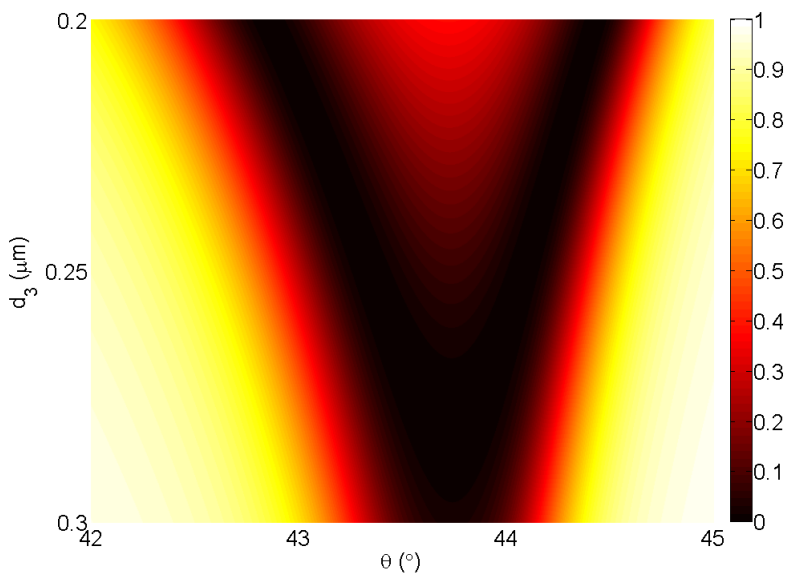

Figure 2. Intensity reflection as a function of angle of incidence and the spacer layer thickness showing the dispersion characteristics of the system under study.

We now look at the response of the structure when it is illuminated by a Gaussian beam. All further calculations were performed with a Gaussian beam with a $50 \mu \mathrm{m}$ waist. Earlier reports have extensively studied the GH shifts and the reflectivity profiles. We do not look into these aspects, concentrating mostly on the reflected beam shape. As mentioned earlier, tightly focused beam undergoes severe distortion in reflection and only few studies exist to deal with such distortions. Very recently, these distortions have been measured [16]. However there are yet no reports that such beam distortions can be used for sensing and other device applications. Here we show that the dip in the distorted reflected beam profile carries information about the resonance in the structure including the attenuated total reflection dip due to the guided mode as well as the sharp phase change at the resonance. Moreover, the location and the spatial location of the dip is extremely sensitive to any asymmetry introduced into the structure. Thus the asymmetry due to different liquids into the sensing channels can be recorded by a displacement and the strength of the dip. Space frequency decomposition method was used for decomposing the Gaussian beam into its various k-components and the reflection coefficient of each $\mathrm{k}$ component was calculated using the transfer matrix formulation [3]. We do not discuss the method in detail since it is now well understood and used by many.

We first present the results for the beam profiles for a symmetric structure where there is no distinction between the forward and backward illuminations. We have investigated all the three regimes, namely, (a) over coupled, (b) critically coupled and (c) under coupled regimes. The results for the modulus of the amplitude as a function of spatial position (with respect to the center of the incident beam) and the angle of incidence are shown through the color plot in Fig. 3. As can be seen from this Fig. 3 (a) due to the two angular dips in the usual reflection profile beam gets maximally distorted at these angles. In contrast, in the critically coupled regime the beam is maximally distorted in the angle range $\left(43.7^{0}\right)$ to $\left(43.8^{0}\right)$ due to the single flat dip in the reflection profile (Fig. 3 (b)). For the under coupled case the reflection dip does not reach zero and hence there is finite reflection even at the resonance locations leading to minimal beam distortion. 

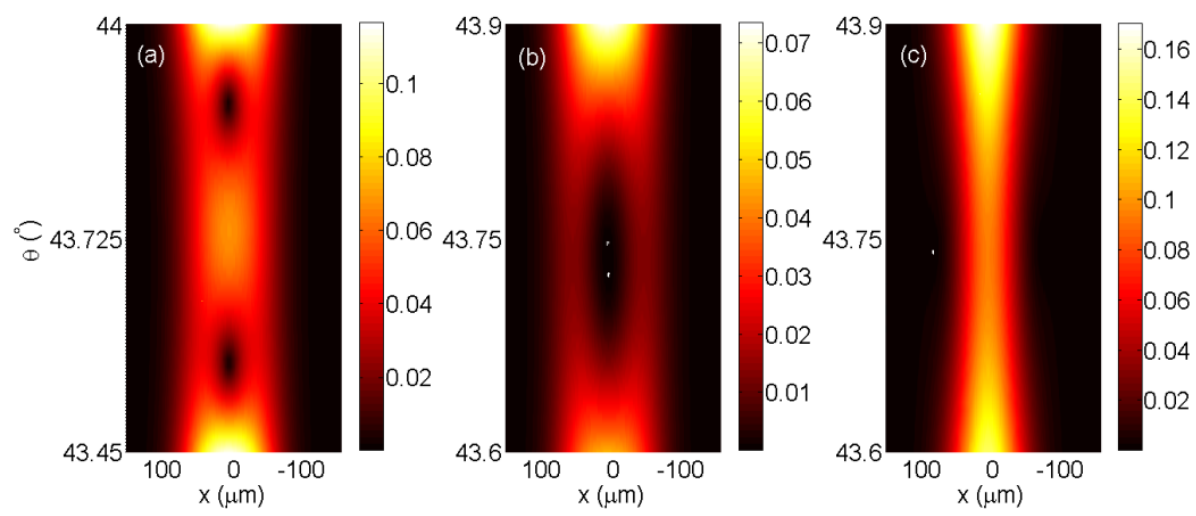

Figure 3. Modulus of the amplitude of the reflected beam as a function of spatial position (from the center of the beam) and the angle of incidence in the (a) over coupled regime when $\mathrm{d}_{3}=0.272 \mu \mathrm{m}$, (b) critically coupled regime when $\mathrm{d}_{3}=0.2795 \mu \mathrm{m}$ and (c) under coupled regime when $d_{3}=0.29 \mu \mathrm{m}$. The incident Gaussian beam has a beam waist of $50 \mu \mathrm{m}$.

In order to study the asymmetric structure we have separated out two cases where there is no absorption and the second one corresponds to a lossy structure (loss mostly from Hemoglobin). In the first case we expect non reciprocal phase with identical amplitude reflection for illumination from opposite ends. Non-reciprocal phase is known to lead to non identical GH shift [9]. Here we show that beam profile as well exhibits this non-reciprocity. In other words one ends up with non identical beam profiles for forward and backward illuminations. The location of the dip in the beam profile is sensitive to the refractive index of the sample liquid while the other reference channel is held fixed. The beam profiles for a structure with water in one channel and a liquid with varying refractive index in the sample channel is shown in Fig. 4. As can be seen from Fig. 4, the reflected beam is no longer Gaussian in the over coupled case for $\mathrm{d}_{3}=0.2795 \mu \mathrm{m}$ and $50 \mu \mathrm{m}$ beam waist. The distortion in the reflected beam disappears for a loosely focused beam (not shown). The minimum (dip) of the distorted Gaussian shifts in opposite directions by -63.02 and $62.21 \mu \mathrm{m}$ for forward and backward illuminations respectively when the dielectric constant of the sample layer is changed by 0.1 (see the inset in Fig. 4 ).

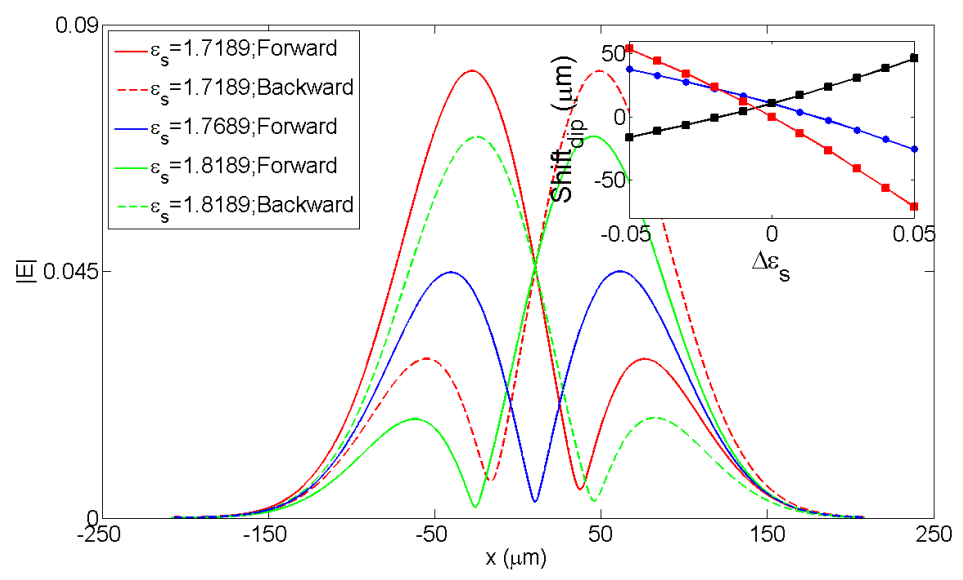

Figure 4. Beam profile change on varying the dielectric constant of the sample layer. The reference sample was taken to be water with dielectric constant $\varepsilon_{\mathrm{w}}=1.7689$.

Now we show that is possible to detect changes in the concentration as well as the fraction of oxygenation of a Hemoglobin $(\mathrm{Hb})$ layer using this configuration. Introduction of Hemoglobin in the sample channel makes the structure lossy. As a consequence there is now non-reciprocity in the reflectivity for opposite illumination. The reference layer is taken as water and the concentration of oxygenated $\mathrm{Hb}$ in the sample layer was varied. It can be seen that on changing the concentration of $\mathrm{Hb}$ in the sample layer from $5 \mathrm{mM}$ to $15 \mathrm{mM}$ ( $\mathrm{Hb}$ concentration in blood varies from 8-15 $\mathrm{mM}$ ) we 
observe a shift of the dip in the beam profile by $-12.63 \mu \mathrm{m}(7.227 \mu \mathrm{m})$ for forward (backward) illumination (see Fig. 5). Note that the change in the oxygenation state of Hemoglobin also leads to shifts albeit smaller in magnitude (not shown).

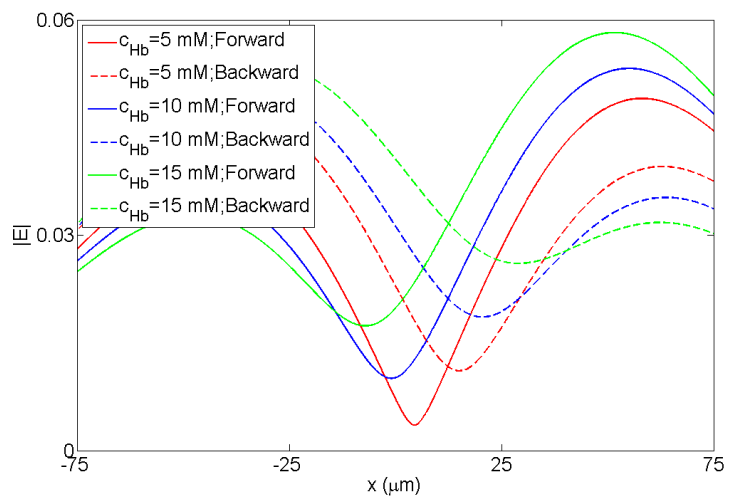

Figure 5. Beam profile modification on varying the concentration of Hemoglobin in the sample layer. The reference layer was taken to be water with dielectric constant $\varepsilon_{\mathrm{w}}=1.7689$.

\section{CONCLUSIONS}

In conclusion we have demonstrated a two channel coupled waveguide system holding great flexibility for refractive index sensing and concentration sensing. The proposed device makes use of the Goos-Hänchen shift and beam distortion under reflection of a tightly focused Gaussian beam.

\section{REFERENCES}

[1] Homola, J., Yee, S. S., and Gauglitz, G., "Surface Plasmon resonance sensors: a review," Sensors and Actuators B: Chemical 54 (1-2), 3-15 (1999).

[2] Brecht, A., and Gauglitz, G., "Optical probes and transducers," Biosensors and Bioelectronics 10 (9-10), 923-936 (1995).

[3] Dutta Gupta, S., "Nonlinear optics of stratified media," in Progress in Optics, E. Wolf, ed. (North Holland, Amsterdam, 1998) 38, pp.1-84.

[4] Yin, X., and Hesselink, L., "Goos-Hänchen shift surface plasmon resonance sensor," Appl. Phys. Lett. 89, 261108 (2006).

[5] Goos, F., and Hänchen, H., " Ein neuer und fundamentaler Versuch zur Totalreflexion," Ann. Phys. (Leipzig) 1, 333-346 (1947).

[6] Artmann, K., " Berechnung der Seitenversetzung des totalreflektierten Strahles," Ann. Phys (Leipzig) 437 (1-2), 87-102 (1948).

[7] Zhao, B., and Gao, L., "Temperature-dependent Goos-Hanchen shift on the interface of metal/dielectric composites," Opt. Exp. 17 (24), 21433-21441 (2009).

[8] Yu, T., Li, H., Cao, Z., Wang, Y., Shen, Q., and He, Y., "Oscillating wave displacement sensor using the enhanceed Goos-Hanchen effect in a symmetrical metal-cladding optical waveguide," Opt. Lett. 33 (9), 10011003 (2008).

[9] Kumari, M., and Dutta Gupta, S., "Positive and negative Giant Goos-Hänchen shift in a Near-symmetric layered medium for illumination from opposite ends," Opt. Comm. in press (doi:10.1016/j.optcom.2011.10.100)

[10] Golla, D., Deb, S., and Dutta Gupta, S., "The role of absorption and dispersion in resonant tunnelling through a negative index medium," Eur. Phys. J. Appl. Phys. 53 (1), 10702 (2011).

[11]Deb, S., and Dutta Gupta, S., "Absorption and dispersion in metamaterials: feasibility of device applications," Pramana - J. Phys., 75 (5), 837-854, (2010). 
[12] Tomita, S., Yokoyama, T., Yanagi, H., Wood, B., Pendry, J. B., Fujii, M., Hayashi, S., "Resonant photon tunneling via surface plasmon polaritons through one-dimensional metal-dielectric metamaterials," Opt. Exp. 16 (13), 9942-9950 (2008).

[13] Agarwal, G. S., and Dutta Gupta, S., "Reciprocity relations for reflected amplitudes," Optics Letters 27 (14), 1205-1207 (2002).

[14] Rao, M. V. S. C., Dutta Gupta, S. and Agarwal, G. S., "Study of asymmetric multilayered structures by nonreciprocity in phases," J. Opt. B: Quantum Semiclass. Opt. 6 (12), 555-562 (2004).

[15] Golla, D., and Dutta Gupta, S., "Goos-Hänchen shift for higher-order Hermite-Gaussian beams," Pramana 7 (4), 603-612 (2011).

[16] Xiao, P., "Beam reshaping in the occurrence of the Goos-Hänchen shift," JOSA B 28 (8), 1895-1898 (2011).

[17] Dutta-Gupta, S. and Martin, O. J. F., "Strong coupled bio-plasmonic system: Application to oxygen sensing," J. Appl. Phys. 110(4), 044701 (2011). 